\title{
Comparing Blood Sugar Levels Measured by the Glucometer in Healthy and Crushed Fingers to Predict Gangrene in Tehran, Iran
}

\author{
Masoud Yavari, ${ }^{1}$ Seyed Esmail Hassanpour, ${ }^{1}$ Marzieh Naghavi Ravandi, ${ }^{1,}{ }^{*}$ and Feizollah Niazi ${ }^{1}$ \\ ${ }^{1}$ Department of Plastic Surgery, 15 Khordad Educational Hospital, School of Medicine, Shahid Beheshti University of Medical Sciences, Tehran, Iran \\ "Corresponding author: Marzieh Naghavi Ravandi, Department of Plastic Surgery, 15 Khordad Educational Hospital, School of Medicine, Shahid Beheshti University of Medical \\ Sciences, Tehran, Iran. Tel: +98-2188901108, Fax: ++98-2188909193, E-mail: Naghavi72658@gmail.com
}

Received 2016 July 13; Revised 2016 October 25; Accepted 2016 November 03.

\begin{abstract}
Background: Crushed fingers are one of the most common reasons that patients visit the emergency centers for hand surgery, and based on the level of injury, it can cause many disabilities for patients. It is difficult to decide the treatment strategies (amputation, aggressive revascularization, immediate or delayed complex reconstruction and immediate conservative treatment) for crushed fingers.

Objectives: The current study aimed to compare the blood sugar (BS) levels measured by the glucometer in healthy and crushed fingers to predict gangrene in patients referred to 15 Khordad Hospital in Tehran, Iran.

Methods: This cohort study was conducted on 265 patients with crushed fingers referred to the emergency center of 15 Khordad hospital in Tehran, Iran, from March 2015 to March 2016. Blood glucose levels were measured by glucometer in the crushed fingers and in the finger of the opposite side at the same time and measurements were recorded. Data were analyzed using t-test and chisquare test with SPSS software version 22.

Results: The results showed that 317 crushed fingers of 265 patients were ischemic based on the color, temperature, capillary refill time and pulse oximetry and accordingly the vascular reconstruction was not possible. Of 317 crushed fingers, 61 (19.24\%) became gangrene (all with sugar levels lower than 37). The mean BS levels of the amputated and non-amputated fingers were $33.5 \pm 1.52$ and $111.04 \pm 15.27 \mathrm{mg} / \mathrm{dL}$, respectively. Therefore, there was a significant difference in the mean BS level between the patients with amputated and non-amputated fingers $(\mathrm{P}<0.001)$.

Conclusions: The lower levels of sugar in crushed fingers compared to healthy fingers can help to diagnose gangrene in crushed fingers.
\end{abstract}

Keywords: Ischemic, Gangrene, Blood Sugar, Glucometer

\section{Background}

Crush injuries of hands and fingers are one of the most challenging categories of the emergency with regards to treatment planning and restoration techniques, especially when it is leading to ischemia. In many cases, given the severity and extent of damage, if amputation is not performed immediately, only minor debridement and wound cleaning, culturing the wound, antibiotics and tetanus prophylaxis, reduction of pieces, examination of the wound, skin restoration, keeping the limbs up and motionless will be performed. In the case of gangrene, amputation and otherwise delayed reconstruction is performed, which is costly and the patient may be at risk of infection (1-3).

Obviously, based on age, occupations, patient's motivation, cigarette smoking, examining functionality of the finger and depth of the injury, if gangrene could be identified with high confidence, an immediate amputation might be beneficial for the patient (instead of conservative treatment). The most important system to examine hand and fingers is the vascular system, which considers the colors of fingers, finger temperature and capillary refill time (CRT) and the diagnostic tools such as pulse oximeter (4).

Metabolic changes occur after ischemia and cellular damage. Anaerobic metabolism is accompanied by reduced oxygen-glucose and adenosine tri-phosphate levels and if the occurrence of damage symptoms prolongs, Co2 and lactic acid levels increase $(5,6)$.

On the other hand, in systemic circulation, glucagon and growth hormone of hyper-glycaemia are produced due to trauma and increase of $\operatorname{cortisol}(7,8)$.

In crushed fingers, glucose reduction occurs due to arterial injury that leads to lack of blood supply or reduced blood circulation in the finger $(5,6)$. On the other hand, increase in the glucose levels in the systemic circulation occurs due to trauma $(7,8)$ and a sugar difference is expected in capillary measured by a glucometer in crushed and healthy fingers and the current study used it to predict gangrene. 


\section{Objectives}

The current study aimed to compare the BS levels measured by the glucometer in healthy (the control group) and crushed fingers to predict gangrene in patients referred to 15 Khordad Hospital in Tehran, Iran.

\section{Methods}

\subsection{The study Samples}

The current study was approved under the registration number of 204 and ethical code of IR.Spmu.sm.REC.1394.142 in Shahid Beheshti University, Tehran, Iran. A pilot study was initially conducted on 20 patients and then from 3110 crushed fingers of 2766 patients referred to the emergency ward of 15 Khordad Hospital of reconstructive and plastic surgery in Tehran, Iran, from March 2015 to March 2016, 317 fingers (of 265 patients) had crushed injuries between wrist fold and distal interphalangeal joint (DIP). Through physical examination of color (dark or pale), temperature (cold) and return CRT greater than three seconds (CRT $>3 \mathrm{~s}$ ), it was determined that patients' finger was suspected for ischemia.

\subsection{Exclusion Criteria}

Cases with obvious gangrene at the registration, and patients with diabetes, kidney and cardiovascular diseases, and connective tissue diseases, those with a history of betablockers, calcium channel blockers and with a history of medications that affect the blood supply and those who were not able to quit cigarettes and hookahs were excluded from the study. Then, the patients were informed about the study objectives and they signed an informed consent form.

\subsection{Procedure}

The patients were taken to the operating room when the damage was so severe and revascularization was not possible. Questionnaires consisting of information on age, gender, finger color, temperature, CRT, $\mathrm{O}_{2}$ saturation and BS measured in the crushed finger ( $s$ ) were completed by trained personnel. The patient was calm and pain free (without local anesthetic and tourniquet) without doing something that cause error in measuring the BS with a glucometer. The BS levels of crushed and healthy fingers (the control group) were measured using the Emperor device made in South Korea, and recorded in the questionnaires while the surgeons and researchers were blinded to the information. All glucometer strips were intact with the same production date, and none of them were expired. Local wound exploration was performed only to determine the situation of tendons; removal was done in cases with an external object and minor debridement was also performed. Because the limb was ischemic, bone fixation was not performed. The skin of the finger was restored and splint was taken in functional status (wrist extension: $20^{\circ}$, MP joint flexion: $70^{\circ}$ and IP joint flexion: minor). The first visit was in the first week and the second and third visits were done during the second and third weeks, and the vital situation of the fingers was examined and recorded in the clinical records and a separate form named form II was completed by the doctor. The doctor did not have any information about the BS level before the surgery or the first questionnaire. During the visits, the wound dressing was changed every other day. Gangrene cases were amputated, and in cases with revived fingers, further construction and physical therapy were performed. After the end of the treatment, the information in the questionnaires and second forms were transferred to SPSS 22, and analyzed statistically. The data including age, CRT, temperature, and color were collected; O2sat of crushed fingers was measured using the pulse oximeter. Also, the BS level was measured by the glucometer in crushed and healthy fingers.

\subsection{Statistical Analysis}

Data were analyzed by paired t-test, cross tabs, chisquare and receiver operating curve (ROC) and then compared between the two groups of the patients (patients with an imputation after three weeks and patients with restored fingers). Data were analyzed using the SPSS software version 22 (SPSS Inc, Chicago, Illinois, USA).

\section{Results}

A total of 2766 patients with 3110 crushed fingers were examined during 2015 - 2016 and those who were not willing to continue the study $(\mathrm{n}=21)$ were excluded. Finally, 317 crushed fingers suspected of ischemia from 265 patients (27 females and 238 males) were examined; the percentage of males (89.8\%) was higher as compared to females (10.2\%). Sixty-one cases of gangrene were amputated, from which six fingers belonged to females. The relationship between ischemic criteria and gangrene fingers showed in the Table 1.

The mean BS levels in crushed fingers that were not gangrenous and those that were gangrenous were 111.04 \pm 15.27 and $33.5 \pm 1.52$, respectively and in the control group, the mean BS levels were $117.73 \pm 20.59$ and $108.38 \pm 14.81$, respectively (Table 2 ). In acute ischemic fingers, there was a significant difference in the mean BS levels between the patients with amputated and non-amputated fingers $(\mathrm{P}<$ 
0.001). In the current study, the prevalence of gangrene in crushed fingers was not correlated with age and gender $(\mathrm{P}=0.68)$. There was a significant difference in color, CRT, temperature and $\mathrm{O}_{2}$ sat factors between the two groups ( $\mathrm{P}$ $<0.001$ ). The crushed fingers with BS $>37.5 \mathrm{mg} / \mathrm{dL}$ had limited necrosis in soft tissues but were not amputated. Patients with crushed fingers with BS $<37.5 \mathrm{mg} / \mathrm{dL}$ were amputated; this test has $98.4 \%$ sensitivity and $100 \%$ specificity (as shown in Table 3). Regarding other measured BS levels with the glucometer and their relationship with gangrene fingers, sensitivity and specificity are explained in Table 3. Table 4 shows the sensitivity, specificity, positive predictive value (PPV) and negative predictive value (NPV) for ischemia criteria and BS $<37.5$ in the crushed fingers.

\section{Discussion}

In the current study the frequency of crushed fingers was higher in males than in females, which was consistent with those of the studies by Okeke in Nigeria (9), Nulu in Turkey (10) and Forutan in Iran (4). So far, there is no study similar to the current study regarding the BS levels measured by the glucometer in crushed fingers to predict gangrene. In a study by Funk DL et al. (11), relative and absolute contraindications of measured capillary were used to measure the blood glucose of poisoned patients in coma due to environmental vasoconstriction reasons, cold extremities, low blood pressure, inflammation and local edema; results of this study were consistent with those of the current study conducted based on peripheral vascular and finger artery damages, coldness, and local inflammation of damaged fingers and obvious difference between sugar levels of two fingers, especially the crushed fingers that later were gangrenous and amputated. In a study by Yaraghi et al. (12) performed in Isfahan on poisoned patients who were in coma, a comparison of capillary and venous blood samples and also glucose levels measured by the glucometer and laboratory tests showed that venous blood glucose samples measured by the glucometer were acceptable, but capillary blood glucose samples measured by the glucometer from patients in coma were not suggested. In other words, the problem was not due to using the glucometer, but due to changes in capillaries, due to local and inflammatory factors and vascular changes, which was also confirmed by the current study. Devan Juneja et al. (13) compared glucose levels of veins and capillaries in patients in shock who received vasopressor. The difference between venous and capillary sugar levels was more than $20 \%$ in $18 \%$ of the patients. In previous studies, the capillary sugar was not compared with the arterial sugar because they focused on the chronic and systemic diseases and damages were in all fingers. In this study, the capillary blood glucose levels were lower due to the use of vasopressor; in the current study the BS level of fingers that later became gangrenous and had arterial injury was lower. In the study by Seyed Forutan et al. (4), the PPV regarding the color of fingers was $25.8 \%$ and in the current study was $26.18 \%$. In the current study, 60 out of 224 cold fingers became gangrenous (26.78\%); this number was $27.51 \%$ in the study by Seyed Forutan et al. In a study by Seyed Forutan et al. (4) on abnormal pulse oximetry (positive) in patients with crushed fingers, $34.78 \%$ of the fingers became gangrenous and this number in the current study was $26.78 \%$. Due to the sensitivity of capillary blood glucose measured by the glucometer to changes in condition, it is a negative factor to measure and adjust the BS level in patients with diabetes. In this study, we decided to use it as a positive and useful tool to predict the probability of gangrene in crushed fingers using tools such as pulse oximetry and other diagnostic methods.

In the current study, 60 crushed fingers (98.36\%) with the BS levels lower than 37.5 (with $98.4 \%$ sensitivity and $100 \%$ specificity unlike other methods) were amputated. The current study can be effective to inform patients, plan treatments and reduce treatment costs.

\section{Acknowledgments}

The current article was extracted from the dissertation written by Mrs. Marzieh Naghavi Ravandi in school of medicine at Shahid Beheshti University of Medical Sciences, Tehran, Iran (registration number: 204). The authors wish to thank the vice-chancellor for research, faculty of medicine, respected professors and staff of 15 Khordad Hospital in Tehran, Iran.

\section{Footnote}

Authors' Contribution: Marzieh Naghavi Ravandi: study concept and design, acquisition of data and drafting of the manuscript; Masoud Yavari: data collection, data analysis, drafting of the manuscript, and critical revisions of the manuscript for important intellectual content; Seyed Esmail Hassanpour and Feizollah Niazi: data collection and analysis. 
Table 1. Relationship Between Ischemia Criteria and Gangrene Fingers

\begin{tabular}{|c|c|c|}
\hline Ischemia Criteria & Amputation, + & Amputation, \\
\hline \multicolumn{3}{|l|}{ Color (dark or pale) } \\
\hline+ & $61(20.7)$ & $233(79.3)$ \\
\hline- & 0 & $23(100)$ \\
\hline \multicolumn{3}{|l|}{$\mathrm{CRT}>3 \mathrm{~s}$} \\
\hline+ & $61(27.9)$ & $158(72.1)$ \\
\hline- & 0 & $98(100)$ \\
\hline \multicolumn{3}{|l|}{ Lower $\mathrm{O}_{2}$ sat } \\
\hline+ & $61(24.4)$ & $189(75.6)$ \\
\hline- & 0 & $67(100)$ \\
\hline \multicolumn{3}{|l|}{ Temperature (cold) } \\
\hline+ & $60(21.1)$ & $224(78.9)$ \\
\hline- & $1(3)$ & $32(97)$ \\
\hline \multicolumn{3}{|l|}{ CFBS $<37.5 \mathrm{mg} / \mathrm{dL}$} \\
\hline+ & $60(100)$ & 0 \\
\hline- & $1(4)$ & $256(99.6)$ \\
\hline
\end{tabular}

Abbreviations: CRT, capillary refill time; CFBS, crushed finger blood sugar; $\mathrm{O}_{2}$ sat, $\mathrm{O}_{2}$ saturation.

Table 2. Mean Blood Sugar Values in Normal and Crushed Fingers and Their Changes

\begin{tabular}{|c|c|c|c|c|c|}
\hline \multirow[t]{2}{*}{ Finger } & \multirow[t]{2}{*}{$\mathbf{n}$} & \multicolumn{3}{|c|}{ Mean \pm SD } & \multirow[t]{2}{*}{ P Value } \\
\hline & & NFBS & CFBS & Changes & \\
\hline Amputation+ & 61 & $117.73 \pm 20.59$ & $33.5 \pm 1.52$ & $-84.22 \pm 20.49$ & $<0.001$ \\
\hline Amputation- & 256 & $108.38 \pm 14.81$ & $111.04 \pm 15.27$ & $2.65 \pm 6.70$ & $<0.001$ \\
\hline Total & 317 & $110.18 \pm 16.47$ & $96.12 \pm 33.55$ & $-14.06 \pm 35.95$ & $<0.001$ \\
\hline
\end{tabular}

Abbreviations: n, number; NFBS, normal finger blood sugar; CFBS, crushed finger blood sugar.

Table 3. The Relationship Between Sensitivity and Specificity and Crushed Finger Blood Sugar

\begin{tabular}{lcc}
\hline Specificity & Sensitivity & CFBS $^{\mathbf{a}}$ \\
\hline $\mathbf{1 0 0} \%$ & 33.8 & 3.5 \\
$\mathbf{1 0 0} \%$ & 86.9 & 35.5 \\
$\mathbf{1 0 0} \%$ & 96.7 & 36.5 \\
$\mathbf{1 0 0} \%$ & 98.4 & 37.5 \\
\hline
\end{tabular}

Abbreviation: CFBS, crushed finger blood sugar.

${ }^{\mathrm{a}}$ Cut-off point: $\mathrm{BS}<31.5$.

Table 4. Evaluation of the Ischemia Criteria Regarding Five Diagnostic Procedures

\begin{tabular}{|c|c|c|c|c|c|}
\hline & Sensitivity & Specificity & PPV & NPV & Yuden \\
\hline \multicolumn{6}{|l|}{ Ischemia criteria } \\
\hline Color (dark or pale) & 100 & 9 & 20.7 & 100 & 0.09 \\
\hline $\mathrm{CRT}>3 \mathrm{~s}$ & 100 & 38.3 & 27.9 & 100 & 0.383 \\
\hline Temperature (cold) & 98.4 & 12.5 & 21.1 & 97 & 0.109 \\
\hline Lower $\mathrm{O}_{2}$ sat & 100 & 26.2 & 24.4 & 100 & 0.262 \\
\hline CFBS $<37.5 \mathrm{mg} / \mathrm{dL}$ & 98.4 & 100 & 100 & 99.6 & 0.984 \\
\hline
\end{tabular}

Abbreviations: PPV, positive predictive value; $\mathrm{NPV}$, negative predictive value; $\mathrm{CRT}$, capillary refill time; $\mathrm{CFBS}$, crushed finger blood sugar; $\mathrm{O}_{2}$ sat, $\mathrm{O}_{2}$ saturation.

\section{References}

1. Reagan DS, Grundberg AB, Reagan JM. Digital artery damage associ- ated with closed crush injuries. J Hand Surg Br. 2002;27(4):374-7. doi: 10.1054/jhsb.2001.0727. [PubMed: 12162982]. 
2. Pederson WC, Stevanoic M. Reconstruction surgery; Extensive injuries to the upper limb. 17. Philadelphia: Saunder; 2006. pp. 317-49.

3. Moran SL, Strickland J, Shin AY. Upper-extremity mucormycosis infections in immunocompetent patients. JHand Surg Am. 2006;31(7):12015. doi: 10.1016/j.jhsa.2006.03.017. [PubMed: 16945728].

4. Seyed Forotan SK, Rahimian S, Akbari H, Mousavi SJ, Hasani E. A comparison between diagnostic methods of ischemia in prediction of digital gangrene probability in HazrateFateme Hospital. Iran J Surg. 2009;17(1).

5. Cohen BE, Harmon CS, Phizackerley PJ. Glucose metabolism in experimental skin flaps. Plast Reconstr Surg. 1983;71(1):79-86. doi: 10.1097/00006534-198301000-00019. [PubMed: 6849026].

6. Im MJ, Su CT, Hoopes JE, Anthenelli RM. Skin-flap metabolism in rats: oxygen consumption and lactate production. Plast Reconstr Surg. 1983;71(5):685-8. [PubMed: 6340141].

7. Vidal-Puig A, O'Rahilly S. Metabolism. Controlling the glucose factory. Nature. 2001;413(6852):125-6. doi: 10.1038/35093198. [PubMed 11557965].
8. Mueckler M, Thorens B. The SLC2 (GLUT) family of membrane transporters. Mol Aspects Med. 2013;34(2-3):121-38. doi: 10.1016/j.mam.2012.07.001. [PubMed: 23506862].

9. Okeke LI, Dogo D, Ladipo JK, Ajao OG. Crush injuries of the hand. Afr J Med Med Sci. 1993;22(3):69-72. [PubMed: 7839916].

10. Unlu RE, Abaci Unlu E, Orbay H, Sensoz O, Ortak T. [Crush injuries of the hand.]. Ulus Travma Acil Cerrahi Derg. 2005;11(4):324-8. [PubMed: 16341971].

11. Funk DL, Chan L, Lutz N, Verdile VP. Comparison of capillary and venous glucose measurements in healthy volunteers. Prehosp Emerg Care. 2001;5(3):275-7. [PubMed:11446542]

12. Yaraghi A, Mood NE, Dolatabadi LK. Comparison of capillary and venous blood glucose levels using glucometer and laboratory blood glucose level in poisoned patients being in coma. Adv Biomed Res. 2015;4:247. doi: 10.4103/2277-9175.170242. [PubMed: 26693472].

13. Juneja D, Pandey R, Singh O. Comparison between arterial and capillary blood glucose monitoring in patients with shock. EurJIntern Med. 2011;22(3):241-4. doi: 10.1016/j.ejim.2011.01.004. [PubMed: 21570641]. 